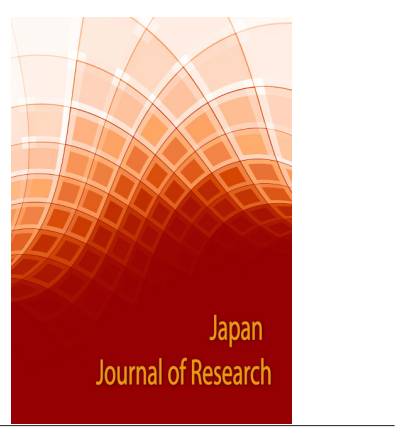

\title{
Predictive control and CSTR application based on step response model
}

\author{
Wei Wang ${ }^{1}$ and Yongjian Sun ${ }^{2 *}$ \\ 'School of Electrical Engineering, University of Jinan, Jinan, Shandong, China. \\ ${ }^{2}$ School of Electrical Engineering, University of Jinan, Jinan, Shandong, China.
}

\section{Correspondence}

Yongjian Sun

School of Electrical Engineering, University of Jinan, Jinan, Shandong, China

E-mail: sunyongjian2006@163.com

\footnotetext{
- Received Date: 15 June 2021

- Accepted Date: 23 June 2021

- Publication Date: 04 Aug 2021
}

Keywords

Prediction model, Online optimization, Control algorithm
Copyright

(c) 2021 Science Excel. This is an openaccess article distributed under the terms of the Creative Commons Attribution 4.0 International license.

\begin{abstract}
Predictive control is a kind of time-domain control method which can display and deal with the control problems of constrained nonlinear systems. It is a new type of computer control algorithm developed in recent years. In the investigation report issued by the International Federation of automatic control in April 19, PID control, system identification, estimation and predictive control after filtering are listed as the most important control technologies, and are considered as the most influential control methods in the future. The inherent robustness of predictive control solves the problem that has puzzled the control theory field for nearly a decade. As soon as it appears, it has been paid attention by the engineering circles at home and abroad, and has been widely and successfully applied in many industrial departments. The current research interests are predictive control, lupon control and the application of predictive control and robust control in electromechanical systems. In the actual industrial production, there is no need for a particularly fine mathematical model, which requires a method that can achieve high quality control effect while the model accuracy is not high First of all, mathematical modelling is needed. The function of prediction model is to predict and control the future output of the system by using the historical information of the controlled object and the assumed future input. Then the optimization algorithm is used for rolling optimization, and the predictive control adopts the finite time domain optimization, which is a kind of repeated online optimization. Last, By detecting the real-time state or output of the current system, the feedback information is used to make the next prediction and optimization closer to the reality before optimizing the control. To avoid the disturbance and system mismatch and other uncertainties, to compensate for the impact of this uncertainty.
\end{abstract}

\section{Introduction}

For predictive control, the research mainly focuses on robustness. When there are requirements for stability performance, stability performance analysis is also the main aspect of research. Industrial production has low requirements for modeling, but the requirements for control are not reduced [1]. Therefore, in the case of modeling error, the analysis of robust performance also needs to be studied. In addition, the research of tracking performance is the optimization of process dynamic response, and the requirement of anti-interference performance is the requirement of system stability. In the study of robustness and stability is not deep enough, in the study of stability when the model parameters are unknown, the model and object can be perfectly matched, and in the optimization analysis of stability, there are not many successful research results. There are also many successful cases of predictive control technology in industrial production, such as predictive control of chemical industry, trajectory guidance of aircraft, predictive control of industrial raw material level, which show the application of predictive control in than traditional control, which not only improves the control effect, but also improves the efficiency, and reduces the waste of raw materials, These are not only single variable single output systems, but also nonlinear multivariable systems. These new prediction systems have been widely used [2].

The research on nonlinear system is not enough, but the chemical industry, machine control and other aspects still have higher requirements for nonlinear system. In the future, the requirements for control accuracy and variables will be increasing. Therefore, the research on nonlinear multivariable system is very important for control, Therefore, the research of nonlinear predictive control technology will be a very important direction in the field of control. At present, it is mainly aimed at some special mathematical models, such as step response, impulse response and so on. There is no general nonlinear predictive control method [3]. Model predictive control is a kind of optimal control strategy based on mathematical model, which can add feedback correction closed loop. It is widely used in chemical industry. practice, and make the system get better effect 
This paper mainly establishes the mathematical model of step response, which does not need the specific parameters of the control object, but needs the state variables and past input and output parameters of the object, Without the complex mathematical analysis of system identification, the optimal control of the system can be carried out, so as to effectively overcome the uncertainty interference and error of the system, so as to achieve the specific requirements. And model predictive control is suitable for multivariable systems because it does not need decoupling, and can directly con- sider various constraints [4]. In the process industry, MPC becomes an algorithm to deal with complex multivariable constraint problems, which is an essential control technology. For more than ten years, as a new control method, predictive control has achieved successful application examples with continuous improvement of algorithm and theory [5]. At present, predictive control has developed into a variety of new predictive control technologies which can be applied to nonlinear variable and multivariable, and has been successfully applied in various fields, The predictive control of reentry guidance of aerospace vehicle, nonlinear continuous predictive control of robot, multivariable coordinated predictive control of mixed constraint process [6].

Multi model generalized predictive control of liquid level system and so on all show the advantages of predictive control algorithm in practical application, which makes the system get good improvement, saves energy and improves efficiency. It also brings huge profits to the enterprise [7]. The development of predictive control is closely related to practical application. At present, qualitative research is more than quantitative research, especially for the analysis of stability and robustness; it is difficult to have the conclusion of relevant analytical relationship [8]. Furthermore, there is no general principle on how to solve the relationship between parameter design and systematisms, breaking through the bottleneck of quantitative analysis is still a research direction in the future [9]. At present, most of the related re- search is linear predictive control, but the research of non- linear predictive control is less. Because the actual industrial process is mostly nonlinear system, the scientific predictive control strategy should be better than linear predictive control. Therefore, the predictive control of nonlinear system is very important, because rolling optimization and feedback correction are based on it [10].

At present, it is mainly for some special models, and there is no general nonlinear predictive control method [11]. Therefore, the theory and application of nonlinear predictive control will be an important direction of predictive control in the future[12].In recent years, the research of predictive control theory mainly analyzes the robustness and stability of the existing predictive control algorithms, and puts forward some new algorithms based on the analysis[13]. When there are some modeling errors in robustness analysis, scholars have conducted in-depth research and discus- sion, but there are too few research results in quantitative description of substantive areas [14].

There are still some problems about the factors affecting the predictive control, especially the influence of the design parameters on the robust stability.

\section{CSTR mechanism model and simulation}

\section{Methods and characteristics of process modelling}

Process modeling mainly includes organic modeling, testing modeling and hybrid modeling. In the continuous stirred reactor model, we mainly use mechanism modeling. The mathematical model will be analyzed and studied in depth to facilitate the simulation and get the corresponding conclusions, and provide more accurate data and guidance for the actual

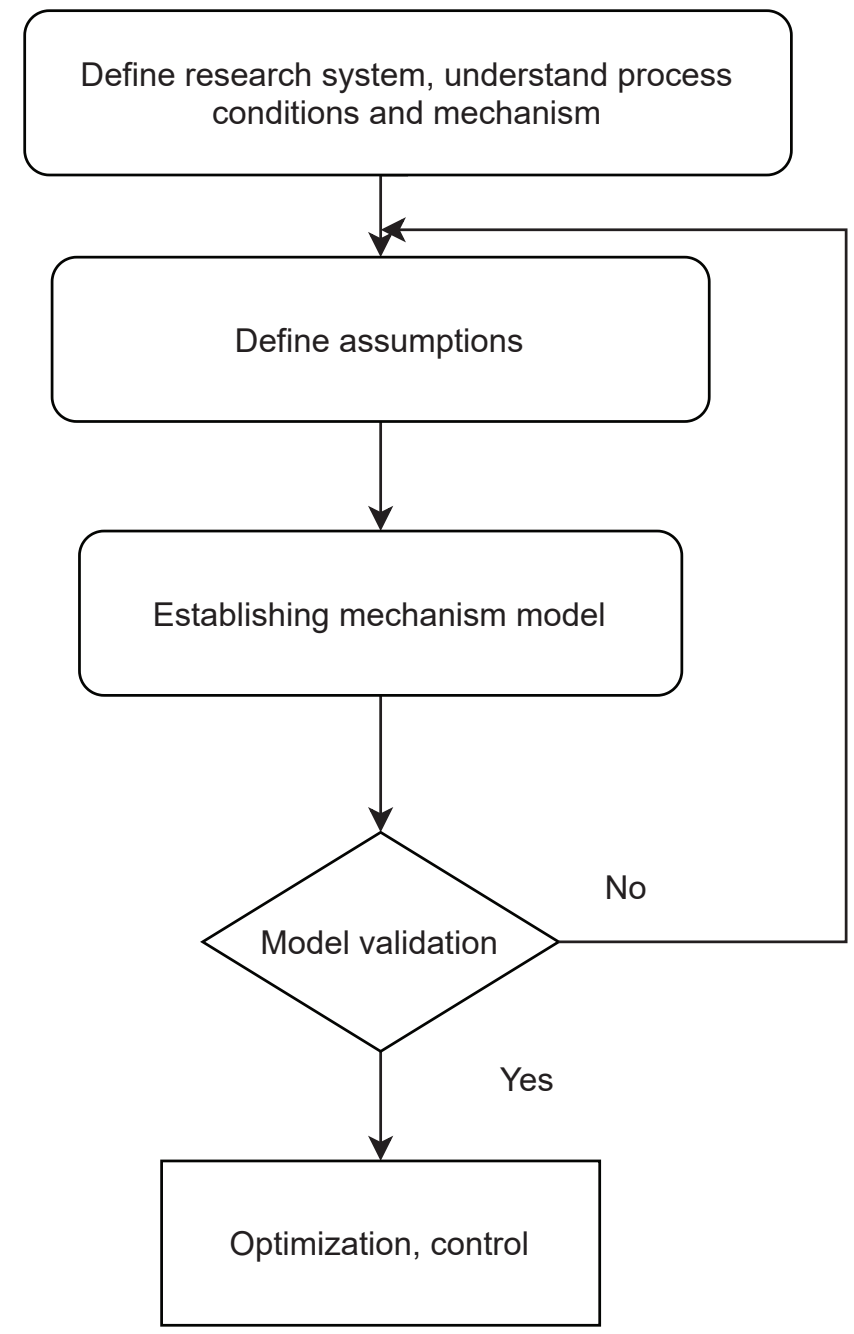

Figure 1: Steps of mechanism modeling.

production. Learning the basic principles of predictive control, MPC based on step response model, mainly includes the research of predictive control algorithm (dynamic matrix control algorithm), parameter design of single variable predictive control system (dynamic matrix control algorithm), parameter design of multivariable predictive control algorithm, and variable predictive control algorithm (dynamic matrix control algorithm) Decoupling of multivariable predictive control algorithm, generalized MPC, GPC. This paper analyzes the main problems of predictive controller algorithm performance to be solved under the influence of different parameters, how to design some parameters of a variable predictive control algorithm, how to decouple the multivariable measurement and control algorithm, and uses MATLAB to simulate the CSTR mechanism model, and verifies the control effect of the algorithm according to the simulation results.

The steps of mechanism modeling are shown in figure 1 .

1. Define the research system, understand the process conditions and mechanism, understand the input and output of the state and various process parameters.

2. The assumption conditions are defined, the unimportant parameters are ignored, and the values of small changes 
are fixed constants.

3. In this paper, the mechanism modeling is mainly based on material conservation, energy conservation and various chemical and physical equilibrium relations to write the basic reaction equation.

4. Finally, the model needs to be tested, and computer simulation is needed to compare the simulation data with the actual data to verify whether the model is in line with the actual situation.

Mechanism modeling of continuous stirred reactor (CSTR)

Continuous stirred reactor is a common reaction device in chemical industry, as shown in figure 2. Given conditions: the input liquid is single component $A$, and its concentration is $C_{A i}$, the flow is $F_{i}$. The temperature is $T_{i}$. At the outlet, the concentration of component $\mathrm{A}$ is $C_{A}$. The concentration of component $B$ is $C_{B}$. The output flow is $F_{o}$, temperature $T$. A cooling jacket is installed outside the reactor, and the coolant flow inside is $F_{c}$. The input temperature is $T_{c i}$ and the output temperature is $T_{c}$.

According to the conservation equation of the material, it is concluded that:

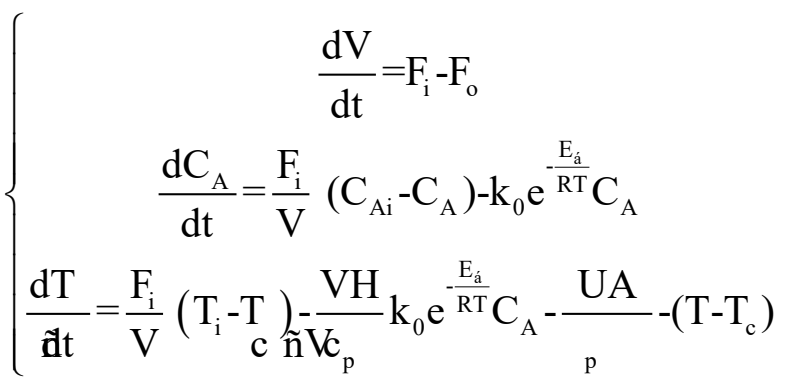

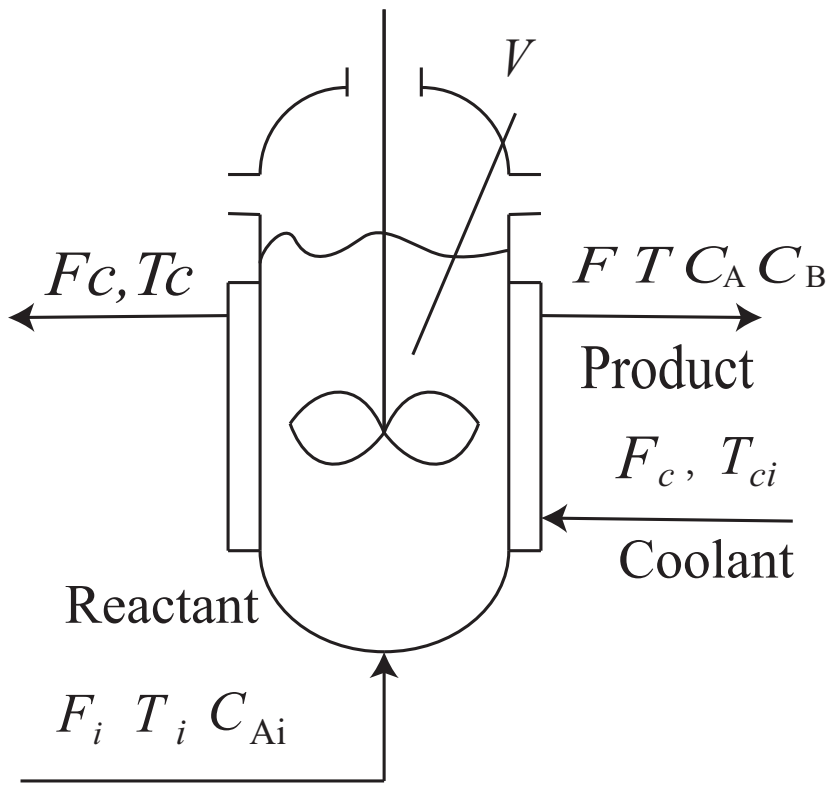

Figure 2: Continuous stirred reactor
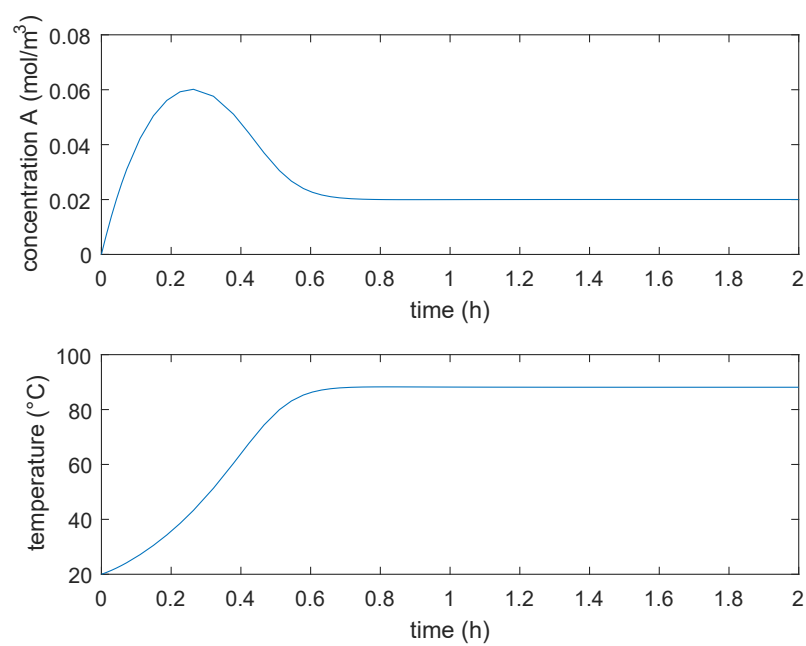

Figure 3: Dynamic response curve of CSTR from initial state to steady state

Simulation and steady point analysis

Figure 3 horizontal axis time, the first ordinate is the concentration of component $A$, and the second ordinate is the temperature of the reactor. It can be seen from the figure that the peak time of component $\mathrm{A}$ is 0.3 hours, the concentration is $0.06 \mathrm{~mol} / \mathrm{m}^{3}$, the steady-state concentration is $0.02 \mathrm{~mol} / \mathrm{m}^{3}$, at 0.7 hours, the overshoot is $200 \%$, and the reactor temperature reaches steady-state at 0.6 hours, that is, the regulation time is about 0.6 hours.

\section{Model prediction algorithm based on step response}

Brief introduction of MPC

If $r$ is the set value and $y^{\wedge}$ is the predicted value of the model, the sum of the square of the residual error between the set value and the predicted value at $k+j$ is obtained, and the cumulative deviation in the prediction time domain is obtained The final objective function is the sum of $\mathrm{u}$-weighted coefficients.

$$
\begin{aligned}
& \Delta u(k){ }_{\Delta U}^{\min }\left(k+N_{c}\right)-1^{J}=\min \left\{\sum_{j=1}^{N_{p}}(r(k+j)-\hat{y}(k+j))^{2}\right. \\
& +\sum_{i=1}^{N_{c}} w_{j}\left(\Delta u(k+j-1(k+j))^{2}\right\}
\end{aligned}
$$

Where NP is the prediction time domain length, which can be written as $\mathrm{P}, \mathrm{NC}$ is the control time domain length, which can be written as $\mathrm{M}$. The above formula is written as a matrix.

$$
{ }_{\Delta U(k)}^{\min }=\min \left\{\| R(k)-\left(\hat{Y}\left\|^{2}+\right\| \Delta U(k) \|_{W}^{2}\right.\right.
$$

\section{Step response model}

When the jacket temperature $\mathrm{Tj}$ is increased by $10 \%$ under the stable condition, the step response curve of the model to the jacket temperature changing with time is obtained. The sampling time is set to one minute. In order to ensure the stable state of the system, that is, 50 sampling points. The step response is shown in figure 4 . 


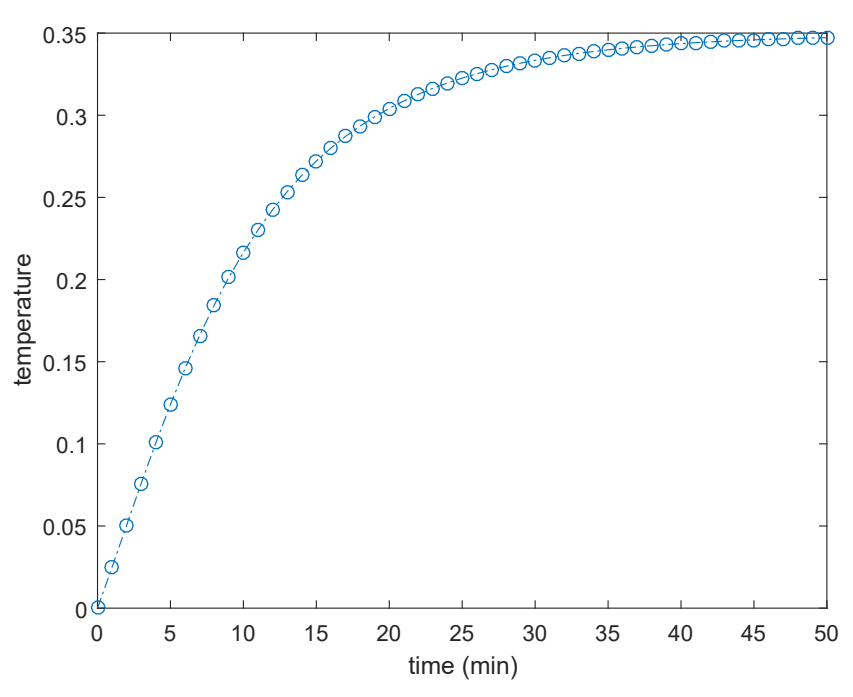

Figure 4: Step response curve of CSTR

Take $\mathrm{S}=\Delta \mathrm{y} / \Delta \mathrm{U}$ to get the step response model.

$$
S=\left[\begin{array}{ll}
S_{1} & S_{2} \ldots S_{N}
\end{array}\right]^{2}
$$

Unconstrained MPC and optimization formula

Step response model predictive control (DMC), also known as dynamic matrix control, predicts the future re- sponse of the system through the existing information, the past input and output of the system and the current state and input of the system. It can be predicted by the current initial value $\mathrm{Y}^{\wedge} 0(\mathrm{k})$. And $\mathrm{M}$ incremental sequences $\Delta \mathrm{U}(\mathrm{k})$ is multiplied by the dynamic matrix a composed of step response sequence.

$$
\hat{Y}(k)=\hat{Y}_{0}(\mathrm{k})+\mathrm{A} \Delta \mathrm{U}(\mathrm{k})
$$

The increment after $\mathrm{s}$ is 0 . Then, the predicted output values from $\mathrm{k}+1$ to $\mathrm{k}+\mathrm{p}$ can be obtained:

$$
\begin{gathered}
\hat{Y}(\mathrm{k}+1)=\mathrm{s}_{1} \Delta \mathrm{u}(\mathrm{k})+\cdots \mathrm{s}_{N-1} \Delta \mathrm{u}(\mathrm{k}-\mathrm{N}+2) \\
+\mathrm{s}_{N} \mathrm{u}(\mathrm{k}-\mathrm{N}+1) \\
\hat{y}(\mathrm{k}+2)=\mathrm{s}_{1} \Delta \mathrm{u}(\mathrm{k}+1)+\cdots \mathrm{s}_{N-1} \Delta \mathrm{u}(\mathrm{k}-\mathrm{N}+3) \\
+\mathrm{s}_{N} \mathrm{u}(\mathrm{k}-\mathrm{N}+2) \\
\vdots \\
\hat{Y}(\mathrm{k}+\mathrm{P})=\mathrm{s}_{1} \Delta \mathrm{u}(\mathrm{k}+P-1)+\cdots+\mathrm{s}_{p-u} \Delta \mathrm{u}(\mathrm{k}+M) \\
+\mathrm{s}_{\mathrm{p}-\mathrm{M}+1} \Delta \mathrm{u}(\mathrm{k}+\mathrm{M}-1)+ \\
\ldots+S_{p} \Delta \mathrm{u}(\mathrm{k})+\mathrm{s}_{p+1} \Delta \mathrm{u}(\mathrm{k}-1)+\cdots \\
+\mathrm{s}_{\mathrm{N}-1} \Delta \mathrm{u}(\mathrm{k}+\mathrm{P}-\mathrm{N}+1)+\mathrm{s}_{\mathrm{s}}(\mathrm{k}-\mathrm{N}+\mathrm{P})
\end{gathered}
$$

The future time and past time in the predicted output value can be classified and written in the form of matrix

$$
\hat{Y}(\mathrm{k})=\mathrm{S}_{\mathrm{f}} \Delta \mathrm{U}(\mathrm{k})+\mathrm{S}_{\mathrm{p}} \Delta \mathrm{U}_{\mathrm{p}}(\mathrm{k})+\mathrm{s}_{\mathrm{N}} \mathrm{U}_{\mathrm{p}}(\mathrm{k})
$$

\section{Introducing feedback correction}

In order to improve the accuracy of the system and reduce the control error caused by nonlinearity and interference, feedback correction is introduced. Feedback correction is a kind of closedloop correction, which makes then output of the system have a direct impact on the system and can increase the accuracy of the system. When the output of the system deviates, it can weaken or offset this part of the error in time. The error here refers to the difference between the measured value and the expected value. In model predictive control, it is the error between the actual measured value and the model predicted value:

$$
\mathrm{d}(\mathrm{k})=\mathrm{y}(\mathrm{k})-\hat{y}(\mathrm{k})
$$

The actual value in the future is unknown. The error with the predicted value of the model cannot be defined. The current error can be brought into the future error, and the error from $k+1$ to $\mathrm{k}+\mathrm{p}$ is equal to the error at $\mathrm{k}$ time. After error correction, the predicted value can be written as:

$\hat{y}^{\mathrm{c}}(\mathrm{k}+\mathrm{j})=\hat{y}(\mathrm{k}+\mathrm{j})+\mathrm{d}(\mathrm{k}+\mathrm{j})$

Combined with eq. ??, we can get:

$$
\begin{gathered}
\mathrm{J}(\mathrm{k})=\left[\mathrm{E}(\mathrm{k})-\mathrm{S}_{\mathrm{f}} \Delta \mathrm{U}(\mathrm{k})\right]^{\mathrm{T}}\left[\mathrm{E}(\mathrm{k})-\mathrm{S}_{\mathrm{f}} \Delta \mathrm{U}(\mathrm{k})\right] \\
+[\Delta \mathrm{U}(\mathrm{k})]^{\mathrm{T}} \mathrm{W}[\Delta \mathrm{U}(\mathrm{k})] \\
=\mathrm{E}^{\mathrm{T}} \mathrm{E}-2 \Delta \mathrm{U}^{\mathrm{T}} \mathrm{S}_{\mathrm{f}}^{T} \mathrm{E}+\Delta \mathrm{U}^{\mathrm{T}}\left[\mathrm{S}_{\mathrm{f}}^{T} \mathrm{~S}_{\mathrm{f}}+\mathrm{W}\right] \Delta \mathrm{U}
\end{gathered}
$$

In order to obtain the analytic solution of the optimal control, we can take the minimization function $J(k)$ pair The first derivative of $\Delta \mathrm{U}$. If you set it to $\frac{d J}{d U}=0$, you can get it $\Delta \mathrm{U}$; The analytic solution of the optimal control vector of $\mathrm{u}$ is as follows:

$$
\Delta \mathrm{U}^{*}(\mathrm{k})=\left[\mathrm{S}_{\mathrm{f}}^{T} \mathrm{~S}_{\mathrm{f}}+\mathrm{W}\right]^{-1} \mathrm{~S}_{\mathrm{f}}^{T} \mathrm{E}(\mathrm{k})=\mathrm{KE}(\mathrm{k})
$$

Take the first term, the optimal control variable of time $\Delta \mathrm{U}(\mathrm{k})$ is used as the predictive control.

\section{Constrained MPC}

In real production, the control quantity of input quantity will be limited to a certain extent, so the optimization problem of DMC after constraint variables should also be considered, including the constraint of control variable $U$, the constraint of control variable change $\Delta \mathrm{u}$, and the constraint of model prediction value ${ }^{\wedge} \mathrm{yc}$.

\section{Restriction of control quantity}

Set the constraints of control variables and the change amount of control variables, that is, set their maximum and minimum values:

$$
\begin{array}{r}
\mathrm{u}_{\text {min }} \leq \mathrm{u}_{\mathrm{k}+\mathrm{j}} \leq \mathrm{u}_{\max } \\
\Delta \mathrm{u}_{\min } \leq \Delta \mathrm{u}_{\mathrm{k}+\mathrm{j}} \leq \Delta \mathrm{u}_{\max }
\end{array}
$$

By analogy, $\mathrm{u}_{\mathrm{K}}$ to $\mathrm{u}_{\mathrm{K}+\mathrm{m}-1}$ in eq. 12 can be expressed by $\Delta \mathrm{u}$, and written as matrix as follows: 


$$
\begin{aligned}
& {\left[\begin{array}{c}
u_{\min } \\
u_{\min } \\
\vdots \\
u_{\min }
\end{array}\right] \leq\left[\begin{array}{c}
u_{k-1} \\
u_{k-1} \\
\vdots \\
u_{k-1}
\end{array}\right]+u_{k-1}\left[\begin{array}{ccccc}
1 & 0 & 0 & \cdots & 0 \\
1 & 1 & 0 & \cdots & 0 \\
\vdots & \vdots & \vdots & \ddots & \vdots \\
1 & 1 & 1 & \cdots & 1
\end{array}\right]\left[\begin{array}{c}
\Delta u_{k} \\
\Delta u_{k+1} \\
\vdots \\
\Delta u_{k+m+1}
\end{array}\right] } \\
& \leq\left[\begin{array}{c}
u_{\max } \\
u_{\max } \\
\vdots \\
u_{\max }
\end{array}\right]
\end{aligned}
$$$$
I_{e x}=\left[\begin{array}{ccccc}
1 & 0 & 0 & \cdots & 0 \\
1 & 1 & 0 & \cdots & 0 \\
\vdots & \vdots & \vdots & \ddots & \vdots \\
1 & 1 & 1 & \cdots & 1
\end{array}\right]
$$

In summary, we can get the following formula:

$$
\left\{\begin{array}{l}
-I_{e x} \Delta \mathrm{U}(\mathrm{k}) \leq U_{k-1}-U_{\text {min }} \\
I_{e x} \Delta \mathrm{U}(\mathrm{k}) \leq U_{\max }-U_{k-1}
\end{array}\right.
$$

\section{Constraints on predicted values}

Set the maximum and minimum value of the predicted value of the model value:

$$
\mathrm{y}_{\min } \leq \mathrm{y}_{\mathrm{k}+\mathrm{j}}^{c} \leq \mathrm{y}_{\max }
$$

From equations 7 and 9, the predicted values of the model are as follows:

$$
\mathrm{Y}_{\min } \leq \mathrm{S}_{\mathrm{f}} \Delta \mathrm{U}_{\mathrm{p}}(\mathrm{k})+\mathrm{S}_{\mathrm{p}} \Delta \mathrm{U}_{\mathrm{p}} \mathrm{k}+\mathrm{s}_{\mathrm{N}} \mathrm{U}_{\mathrm{p}}(\mathrm{k})+\mathrm{D}(\mathrm{K}) \leq \mathrm{Y}_{\max }
$$

The past moment and error are abbreviated as:

$$
\mathrm{F}(\mathrm{k})=\mathrm{S}_{\mathrm{p}} \Delta \mathrm{U}_{\mathrm{p}} \mathrm{k}+\mathrm{s}_{\mathrm{N}} \mathrm{U}_{\mathrm{p}}(\mathrm{k})+\mathrm{D}(\mathrm{K})
$$

It can be converted into a constraint form of k-time control increment $\Delta \mathrm{U}$ :

$$
\begin{aligned}
& -\mathrm{S}_{\mathrm{f}} \Delta \mathrm{U}(\mathrm{k}) \leq \mathrm{F}(\mathrm{k})-\mathrm{Y}_{\text {min }} \\
& \mathrm{S}_{\mathrm{f}} \Delta \mathrm{U}(\mathrm{k}) \leq \mathrm{Y}_{\text {max }}-\mathrm{F}(\mathrm{k})
\end{aligned}
$$

The optimization function $J$ is written as quadratic form, and the above constraints are added, namely, DMC algorithm with constraints, also known as QDMC algorithm form:

$$
\begin{gathered}
\min _{\Delta u(k)} J(\mathrm{k})=\min _{\Delta u(k)} \frac{1}{2}[\Delta \mathrm{U}(\mathrm{k})]^{\mathrm{T}} \mathrm{H}[\Delta \mathrm{U}(\mathrm{k})]+[\mathrm{C}(\mathrm{k})]^{\mathrm{T}}[\Delta \mathrm{U}(\mathrm{k})] \\
\text { s.t.A } \Delta \mathrm{U}(\mathrm{k}) \leq \mathrm{B} \\
\Delta \mathrm{U}_{\text {min }} \leq \mathrm{U}(\mathrm{k}) \leq \Delta \mathrm{U}_{\text {max }}
\end{gathered}
$$

\section{Matlab simulation}

\section{Unconstrained DMC simulation}

Using the model instead of the actual equipment set the prediction time domain length $\mathrm{P}$, control the time domain length $\mathrm{m}$ and weight $\mathrm{W}$ to 10,2 and 1 respectively, and get the simulation results as shown in figure 5 .
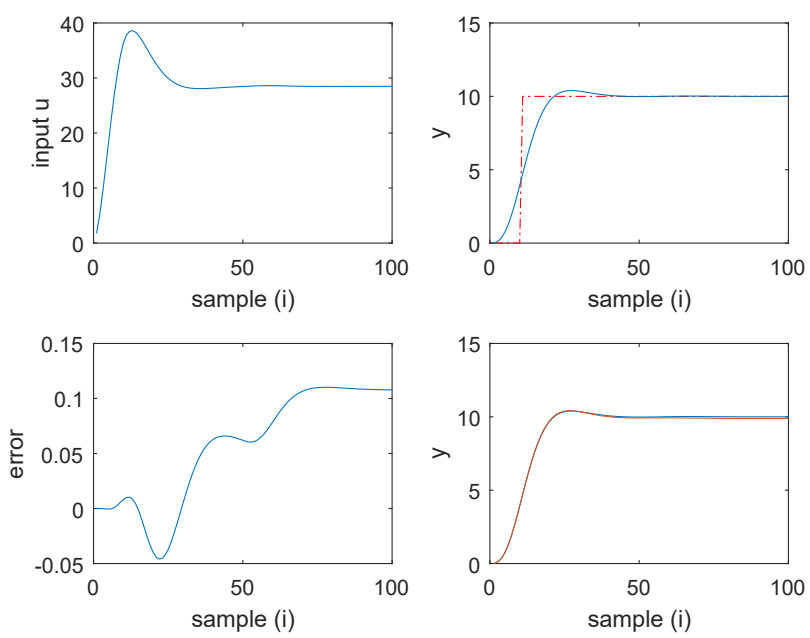

Figure 5: Unconstrained step response curve

It can be seen from the figure above that the overshoot of the system response curve is generated, and it is stable when the abscissa is 40 , that is, the sampling time is 40 . In the predictive control, the sampling theorem should conform to the Shannon sampling theorem satisfied by the control system, so that the value of the sampling frequency should be greater than twice the maximum frequency of the sampling signal. For the model parameters, the upper limit of prediction time domain $\mathrm{P}$ can be selected according to the sampling period $\mathrm{T}$. When the sampling time is small, the length of prediction time domain $\mathrm{P}$ and the length of control time domain $\mathrm{m}$ need to be increased. If we only consider the influence of $\mathrm{P}$ and $\mathrm{m}$ on the system, the prediction time domain $\mathrm{P}$ mainly affects the rapidity and stability, while the control time domain m mainly affects the dynamic performance of the system. When $\mathrm{P}$ is small, the step size of the system is small, and the fast performance is good, but the system has poor anti-interference ability and poor stability; When $\mathrm{P}$ is large, the stability is good, but the system reaction is slow. The magnitude of the control time domain $\mathrm{m}$ should be smaller than the prediction time domain and larger than the non-minimum phase response of the system. When adding noise or disturbance into the program, observe the simulation results as shown in figure 6 . It can be seen that the prediction curve basically coincides with the output curve, which shows that the system has strong suppression 

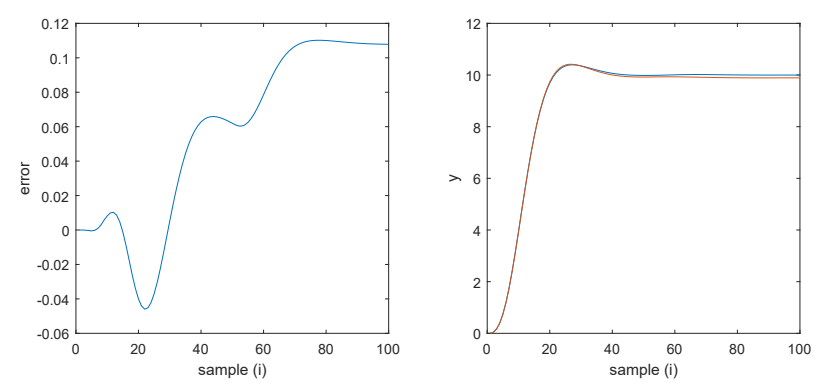

Figure 6: Unconstrained step response curve with disturbance

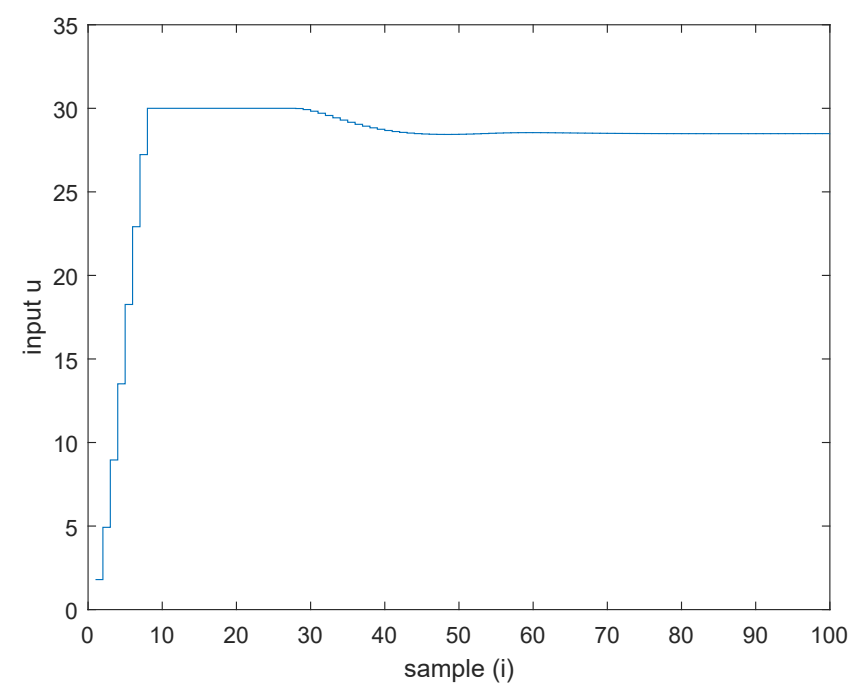

Figure 7: Input maximum suppression curve

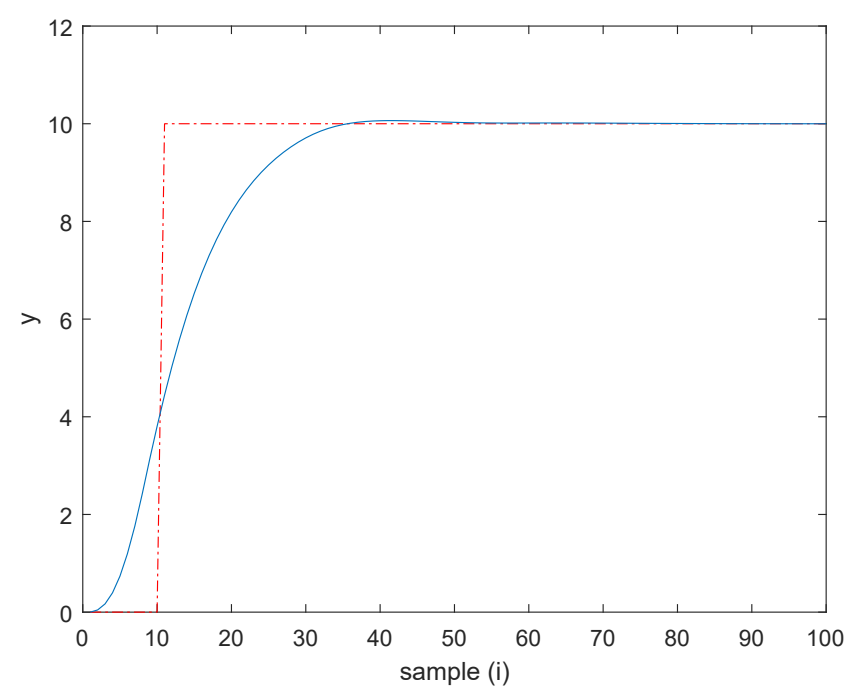

Figure 8: QDMC response curve of interference. When there is interference, the algorithm can also well predict the input and output of the system, and then quickly control the system.

\section{QDMC simulation}

Constraint control is applied to the step response model, where the maximum value of input is limited. Set the maximum value of input $U_{\text {Max }}=30$, as shown in figure.7, compared with unconstrained input, the maximum value of unconstrained input is limited below 30, which suppresses excessive disturbance at the input level.

According to the QDMC response curve in figure 8, compared with the unconstrained system, the algorithm can effectively suppress the overshoot and improve the speed of the system.

\section{Conclusions}

As a new control algorithm, predictive control does not need accurate model structure and parameters, but according to the existing knowledge, in the known information, according to the requirements of the principle of predictive control, simple modeling, and has better control characteristics, so it is widely used in various industries, compared with the traditional PID control, The online optimization feature of predictive control can always optimize the current data online, and is more suitable for the influence of noise and interference in industrial production. Compared with PID, parameter tuning is more convenient and robust. In the comparison between unconstrained MPC and QMPC, it can be seen that the step response model prediction algorithm with constraints has better suppression of overshoot, can effectively restrain the influence of interference on the system, and can more accurately predict the future output of the system with constraints, and can effectively optimize the control of the changes of the future system.

\section{References}

1. Cutler CR, Ramaker BL. Dynamic Matrix Control A Computer Control Algorithm. Proceedings of Joint Automatic Control Conference. Piscataway IEEE,1980:WP5-B.

2. Alpbaz M, Karacan S, Cabbar Y, et al. Application of model predictive control and dynamic analysis to a pilot distillation column and experimental verification. Chemical Engineering Journal. 2002; 88(1-3):63-174

3. Brian FJ. Model predictive control-building a bridge between theory and practice with direction dependent dynamic responses. Computers and Chemical Engineering. 2006;30(1012):1426-1435

4. Rouhani R, Mehta RK. Model Algorithmic Control (MAC), Basic theoretical properties. Automatiea.1982;18(4):401-414.

5. Wu B, Luo X, Wei M, Xu F. Analysis on the selection of prediction time domain in the application of predictive control engineering. Chemical automation and instrumentation. 2017;44(6):553-558.

6. Sheng Y, Su H, Chu J. Predictive control for polytopic uncertain linear systems with guaranteed constraints satisfaction. Control Theory Applications, 2003;2.

7. Hou N. Research status and Prospect of predictive control algorithm. Journal of Hubei Radio and TV University. 2013;33(11):157-158.

8. $\mathrm{Hu}$ G, Sun Y. Research progress and application of predictive control. Journal of power system and automation. 2003;5.

9. Li S, Xu X, Li P. A survey of the latest algorithms of predictive control. Journal of gate system simulation. 2004;6.

10. Han C, Liu X. Robust predictive control for uncertain continuous time systems based on output feedback [J].Information and control, 2006;6. 
11. Du X, Xi Y, Li S. Robust predictive control for uncertain continuous time systems based on output feedback. Information and control. 2006;6.

12. Gao Y, Ji B, Bai G. Application of model based predictive control in temperature control. Automation technology and application. $2011 ; 6$.
13. Wang J, Lei Y. Application of fuzzy predictive control in time delay systems. Automation technology and application. 2007;3.

14. Zhang R, Wang S. Predictive functional control for nonlinear systems based on neural network. Control Theory \& Applications, 2007,24(6):949-953. 